\title{
Beschreibungen africanischer Chrysomeliden nebst synonymischen Bemerkungen.
}

Von

J. W e is e.

1. Sagra Kolbei Clavar. Catal. Ann. Belg. 1900, $274=\sigma^{\star}$ longipes Kolbe, Stuhlm. Ostafr. [4] 1897, $324=0$ opaca Jac. Trans. Ent. Soc. $1888,189=\sigma^{\star}$ longefemorata Fairm. Ann. Fr. $1887,347=$ ferox Baly Journ. Linn. Soc. $1877,338$.

2. Sagra sulcipennis Kolbe 1. c. $325=$ striatipennis Jac. Novitates 1894, 108.

Prosternum schmal, hinten im Bogen abfallend, Mesosternum sebr schmal; die muldenförmige Rinne der Hinterschienen beim $\sigma^{\star}$ kabl, oder nur mit vereinzelten aufstehenden bellen Härchen.

3. Sagra tibialis Fairm. Ann. Belg. 1891, C. R. 302, mit metallisch grüner, blauer, kupferrother Oberseite, Mrogoro, Pare Gebirge (v. Bennigsen), Mombas (Schneider) halte ich nur für eine kleine Form von Cambieri Duviv. Ann. Belg. 1889, C. R. 128, Usegua, Usagara (v. Bennigsen), Mombas (Schneider), bei welcher der grofse Seitenzahn der Hintertibien des $\sigma^{\pi}$ in eine kleine, unscheinbare, zahnförmige Erweiterung des Aufsenrandes reducirt, zuletzt völlig geschwunden ist.

4. Pedrillia Kwaiensis: Elongata, testaceo-rufa, ochraceopubescens, nitidula, antennis (articulis tribus primis exceptis) piceis, mandibulis et tibiis apice tarsisque infuscatis, prothorace ante basin marginato, subtilissime punctato, elytris subtiliter punctatis. - Long. $3-3,5$ mill.

Kwai (Paul Weise).

Diese Art muls der P. madagascariensis Jac. Proceed. 1897, 244 sehr ähnlich sein, scheint aber durch feinere Punktirung und die helle Behaarung des Halsscb. abzuweichen. Sie ist hell und lebhaft bräunlich roth, blafs ockergelb behaart, die Schenkel heller, gelbbraun, die $\&$ Endglieder der Fühler pechschwarz und die Spitze der Mandibeln und Schienen nebst den Tarsen etwas angedunkelt. Kopf mit der normalen graden Querrinne zwischen der Füblerwurzel, die Stirn in der Mitte glatt, zu beiden Seiten nicht dicht und sehr fein punktirt. Halsschild wenig breiter als lang, vor der gerundeten Basis mit grader Querrinne, die sich an den Seiten 
verbreitert und vertieft und dadurch die Einschnürung hinter der Mitte hervorruft. Vor dieser erweitern sich die Seiten in starkem Bogen und verengen sich dann allmählich nach vorn. Die Scheibe ist mäfsig dicht, etwas stärker als der Kopf punktirt; die Tastborsten in den Ecken sehr lang. Fld. etwas stärker punktirt als das Halsschild.

Die Stellung der Gattung zu den auch habituell verschiedenen Megalopinen, die Chapuis, Genera 10,88 et 94 , annimmt, ist in jeder Hınsicht verfehlt und stört die Einheitlichkeit der Gruppe, in welcher nur Thiere mit einfachen Klauen vorkommen. Pedrillia, deren Klauen ein zahnförmiges Anhängsel an der Basis besitzen, gehört zu den Zeugophorinen und ist von Zeugophora überhaupt nur durch minimale Unterschiede zu trennen.

\section{Herma n. gen.}

Genere Melitonoma similis, sed prothorace basi medio in lobum acutum productus.

Diese Gattung, die in der Körperform den kleineren MelitonomaArten äbnlich ist, unterscheidet sich von allen andern durch den Hinterrand des ziemlich stark querüber gewölbten Halssch. Derselbe verlängert sich in der Mitte in einen breit dreckigen Fortsatz, der sich auf das Schildchen legt, dessen vorderen, dicht punktirten und behaarten Theil verdeckt und nur die äufserste glatte Spitze frei lälst.

Herma insignis: Subcylindrica, nigra, antennis (apicem versus sensim infuscatis), genubus, tibiis tarsisque rufo-testaceis, prothorace rufo-testaceo, subpolito, scutello nigro apice rufescente, elytris testaceo-flavis, sat crebre punctatis, singulo maculis tribus nigris, prima humerali, rotundata, secunda juxta suturam paullo ante medium, angusta, parva, obliqua, tertia pone medium, magna, transversa. Long. 6 mill.

\section{Mombo (Paul Weise).}

Kopf mälsig grofs, Augen grofs, länger als breit, sehr fein facettirt, ganzrandig, nicht höher gewölbt als die Stirn. Diese breit, ziemlich eben, mit einem sehr schwachen gebogenen Quereindrucke zwischen den Augen. Mandibeln und Taster dunkel rothbraun. Fühler vom 4. Gliede an verbreitet, röthlich gelbbraun, die folgenden Glieder allmählich immer mehr angedunkelt. Halssch. doppelt so breit als lang, lebhaft röthlich gelbbraun, an den Rändern etwas beller, fast glatt, alle Ecken abgerundet, der Fortsatz stark gewölbt. Schildehen an der Spitze verloschen rötblich. Fld. 
mälsig dicht, nicht besonders tief punktirt, an der Spitze fast glatt, mit abgerundeter Nahtecke, hell bräunlich gelb, jede mit drei schwarzen Makeln: die erste mälsig grofs, gerundet, auf und neben dem Schulterböcker, 2 klein, dicht vor der Mitte nahe der Naht, fast doppelt so lang als breit, schräg von vorn nach binteu und innen gerichtet, 3 sehr grofs, vor der Spitze, quer, bindenförmig, aufsen breit, nach innen verschmälert, der Vorderrand schwach gerundet, der Hinterrand in leichtem Bogen ausgeschnitten, innen bis dicht an die Naht reichend, aufsen etwas weiter vom Seitenrande entfernt. Unterseite schwarz, grau behaart, namentlich dicht auf den Seitenstücken der Hinterbrust; Knie, Schienen und Tarsen röthlich gelbbraun.

6. Aspidolopha usambarica $\left.{ }^{1}\right)$ : Subparallela, nigro-cyanea vel obscure coerulea, interdum viridi-micans, subopaca, subtus dense griseo-pubescens, opaca, ore, antennis pedibusque nigris, fronte utrinque juxta oculos crebre punctata et pubescente carinis angulatis evidenter instructa, prothorace parce inaequaliter, latera versus fortius punctato, scutello basi dense punctulato, apice laevi subcarinato, ely tris creberrime, profunde punctatis, interstitiis angustissimis subtilissime punctulato-rugulosis, fere alutaceis. - Long. 4-5 mill.

Mombo (März, August 1899. Paul Weise).

Die Gattung Aspidolopha, mit Diapromorpha nicht näher verwandt, ist durch die Stirnbildung sehr ausgezeichnet. Das Kopfschild und ein mit demselben verbundener dreieckiger Theil der Stirn liegen in einer Ebene. Das Kopfschild ist sparsam behaart, das Stirndreieck darüber kahl, beide werden seitwärts durch zwei Leisten mehr oder weniger scharf begrenzt, welche in der Mitte der Stirn zusammenstofsen, etwas unterhalb der Linie, welche den Oberrand der Augen verbinden würde. Am Innenrande jedes Auges bleibt ein lang dreieckiger behaarter Raum übrig.

1) Eine ähnlich gefärbte Art liegt mir von Manila vor:

Aspidolopha manilensis: Oblongo-ovalis, nigro-coerulea, sat nitida, prothorace subcyaneo, subtus griseo pubescens, subopaca, ore, antennis (articulo primo apice, secundo et tertio omnino rufo-testaceis exceptis) pedibusque nigris, femoribus subaeneis, capite fere laevi, fronte utrinque juxta oculos punctulata et pubescente, prothorace sublaevi punctis nonnullis parvis ante angulos posticos impresso, scutello laeri apice vix carinato, elytris sat dense punctatis, punctis pone medium subtilioribus, ante apicem evanescentibus, interstitiis fere laevibus. - Long. 5 mill. 
7. Corynodes Bennigseni: Elongato-ovatus, splendide castaneus, mandibulis, palpis coxisque nigris, antennis pedibusque nigroviridi-coeruleis, illis clava cyanea, prothorace antrorsum sensim angustato, subcylindrico, parce obsoleteque punctulato, elytris basi prothorace multo-, pone medium duplo latioribus, subtiliter parum profunde punctatis, punctis nigricantibus, unguiculis appendiculatis. Long. 11,5-13,5 mill.

Moa, Afr. or. germ. (v. Bennigsen).

Lefèvre nennt die Klauen seines Coryn. Raffrayi gespalten; sie sind jedoch bei den Stücken von Mrogoro (v. Bennigsen), die ich nach der Beschreibung für diese Art halte, nur unterhalb der Mitte eingeschnitten, wodurch ein stumpfer Basalzahn entsteht, und dürfen daher nicht als bifid bezeichnet werden. Das Halssch. des Raffrayi ist etwas länger als breit, von oben betrachtet in den hinteren $\frac{2}{3}$ allmählich und sehr schwach nach vorn verengt, im letzten Drittel etwas stärker verschmälert, die Seiten convergiren also wenig in einem leichten Bogen. Die Fld. sind vorn kräftig, tief punktirt, mit sehr fein gewirkten $Z$ wischenräumen, mäfsig glänzend, auf dem Abfalle zur Spitze sparsamer und feiner punktirt, aber stärker gewirkt, ziemlich matt. Auf der inneren Hälfte der Scheibe treten oft die Punkte zu vier nicht ganz regelmäfsigen Reihen zusammen, welche 2 Längslinien begrenzen, eine davon in der Mitte, die andere zwischen dieser und der Naht. Die Farbe der Oberseite ist gesättigt bräunlich-roth.

Diesem Thiere ist Coryn. Bennigseni sehr ähnlich, aber oberseits viel feiner und verloschener punktirt, nicht gewirkt, dunkler bräunlich roth gefärbt, stark glänzend, zuweilen mit einem zarten bläulichen oder violetten Anfluge. Das Halssch. ist schlanker, an der Basis etwas schmaler, nach vorn viel mehr verengt, konischcylinderförmig. Die Punkte der Fld. sind klein und sehr flach, jeder steht in einem schwärzlich durchscheinenden Kreise, der wenig gröfser ist als der Punkt selbst.

\section{Palimbola nov. gen. Chrysomelinorum.}

Corpus ovale, alatum, testaceo-rufum, supra sat convexum, punctatissimum, sat nitidum, subtus planum, nitidum. Prothorax fortiter transversus, omnino marginatus, antrorsum rotundatim angustatus, angulis obtusis, posticis pilis nonnullis brevibus instructis. Elytra callo humerali costaque marginali, epipleuris acute inflexis, latis, pone medium sensim angustatis, glabris. Prosternum processu postico brevissimo; acetabula antica aperta. Tibiae apice externe in dentem mag- 
nam productae. Tarsi articulo quarto subtus apice utrinque dentatodilatato. Unguiculi simplici.

Die Körperform, die Gestalt des Halssch. und die Punktirung der Oberseite erinnern an Plagiodera, z. B. an ferrugata Ws., das Thier ist aber neben Melasoma zu rücken, von dem es auf den ersten Blick durch die gezähnten Schienen abweicht. Diese verbreitern sich nach der Spitze hin und sind an dieser in einen sehr grofsen, dreieckigen Zahn ausgezogen, welcher am Ende leicht abgerundet und unterseits zur bequemen Einlagerung der Tarsen ausgeböhlt ist. Eine ähnliche Bildung findet man in Phytodecta und Centroscelis; aber bei ihnen ist die Spitze der Tibien nur zahnartig erweitert, da der Haarkranz nach aufsen bis zur Spitze der Erweiterung reicht, während in Palimbola ein wirklicher, am Vorderrande kahler Zahn vorbanden ist.

Kopf klein, Kopfschild deutlich abgesetzt, Stirn mit weiter und tiefer Mittelrinne. Halsschild mehr als doppelt so breit wie lang, mit bogenförmiger, jederseits sanft ausgeschweifter Basis und scharfen, stumpfwinkeligen Hinterecken, vor diesen anfangs leicht erweitert, vor der Mitte stark verengt, so dafs die Seiten einen Bogen bilden; vorn in gleichmäfsigem Bogen ausgerandet; ringsum gerandet, ohne Borstenporen, nur mit einigen kurzen Härchen neben den Hinterecken. Die Hüften sind grols, die vorderen Hüftgruben weit geöffnet, der Fortsatz des Prosternums sehr kurz, nach hinten erweitert, wenig über die Hüften hinaus ragend, am Ende ausgerandet-abgestutzt, in eine schwache Quergrube der Mittelbrust eingreifend. Die Hinterbrust verlängert sich zwischen die Mittelhüften und bildet einen mälsig breiten, gerundeten und scharf gerandeten Fortsatz. Das zweite Tarsenglied ist kleiner als die einschliefsenden, das dritte an der breiten Spitze wenig ausgerandet, das vierte endet unterseits neben jeder Klaue in einen feinen, spitzen Zahn.

Die Gattung Anomomera Fairm. Ann. Fr. 1887, 360, scheint eine gewisse Aehnlichkeit mit der vorliegenden zu haben, kann jedoch nicht identisch sein, da ihre Klauen „appendiculés, les deux branches grêles, aegues" und die Hinterschenkel "renflés presque autant que chez les Halticides" genannt werden.

Palimbola nigripes: Supra saturate testaceo-rufa, nitidula, subtus nonnihil pallidiore, nitida, mandibulis apice, antennis (articulis tribus primis inferne exceptis) pedibusque nigris, elytris crebre minus fortiter punctatis, punctis septem nigris, 2, 1, $\frac{1}{2}$ collocatis. - Long. 9,2 mill.

Montes Ukami, Mikindani. 
Der Kopf ist dicht punktirt, auf dem Kopfschilde etwas stärker und dichter als auf der Stirn, in der breiten Mittelrinne fast glatt. Halsschild stark querüber gewölbt, mit zahlreichen kleinen und unbestimmten Vertiefungen, in der Mitte der Scheibe sparsamer und feiner, nach den Rändern hin dichter und stärker punktirt. Schildchen eckig-halboval, etwas uneben, fast glatt. Fld. in den Schultern gerundet, heraustretend, dann sehr sanft erweitert, im letzten Drittel schneller gerundet-verengt, mit der höchsten Erhebung vor der Mitte, auf der Scheibe gleichmäfsig, dicht und wenig stark punktirt, mit einzelnen Pünktchen in den schmalen $Z_{\text {wischen- }}$ räumen. Die Schulterbeule und die Längsrippe über dem Seitenrande sind ziemlich glatt, letztere hat aber im äufsersten Theile eine unregelmälsig weitläufige Reihe von groben Punkten, dicht neben der feinen Punktreibe in der Rinne, die den Seitenrand absetzt. Brust (mit Ausnahme der. Seitenstücke) und Bauch sind ziemlich glatt. Beim $\sigma^{\star}$ ist das erste Tarsenglied erweitert, der Hinterrand des fünften Bauchringes in der Mitte schwach abgestutzt und jederseits mit einem Haarbüschel versehen.

Unterseite glänzend röthlich gelbbrann, Beine schwarz, das Prosternum zwischen den Hüften und die Seitenstücke der Hinterbrust oft schwärzlich; Oberseite hell bräunlich roth, mäfsig glänzend, Fld. zusammen mit 7 schwarzen, punktförmigen Makeln, 2 dicht vor $\frac{1}{3}$ der Länge, auf jeder Fld. in einer geraden Querreibe, unter sich etwas weiter entfernt als von der Naht und dem Seitenrande, die dritte dicht vor $\frac{2}{3}$ der Länge fast genau hinter der äufseren Vordermakel, die gemeinschaftliche endlich unmittelbar vor der Spitze.

\section{Ootheca Chap., Genera 11, 173.}

Der Autor behauptet rom Halsechilde: "surface convexe, sans aucune trace d'impression". Dasselbe besitzt jedoch, namentlich in der typischen Art, einen mehr oder weniger langen und tiefen, nach innen gebogenen Längseindruck jederseits, ein Stück über dem Seitenrande. Die Borstenporen in den Ecken sind grofs. Beim Schildchen mufs erwähnt werden, dafs der sehr verdünnte und glasartig durcbscheinende Rand, in den die Seiten und die Spitze verlängert sind und der sich auf den polirten und niedergedrückten Nahtrand der Fld. legt, deutlicher und viel breiter wie bei andern Galeruciden entwickelt ist. Auch die Tibien sind nicht "subcylindriques", sondern haben zum Unterschiede von ähnlichen Gattungen auf dem Rücken eine durchgehende glatte Mittelleiste; die Hüftpfannen der Vorderbeine sind nicht "ouvertes", sondern ge- 
schlossen, da die schmalen Seitenstücke verlängert und mit dem dreieckig erweiterten Ende des Prosternums verbunden sind; endlich trennt das Prosternum die Vorderbüften, da es immer zwischen ihnen sichibar ist. Chapuis nannte dasselbe: „indistinct entre les hanches". Das đَ hat den für die Galeruciden typischen Einschnitt vom Hinterrande des 5. Bauchringes aus jederseits. Derselbe ist nicht besonders lang und begrenzt einen Mittelzipfel, welcher sich zur Mittellinie hin stark vertieft.

Noch schlechter wie die seitherige Begrenzung der Gattung ist die Kenntnifs ihrer Arten. Die vielseitig beschreibenden Entomologen werfen sich hauptsächlich auf die auffallenden, leicht zu unterscheidenden Thiere und gehen einer Untersuchung der sehr ähnlichen Formen möglichst aus dem Wege; sie halten sich noch immer an die veraltete Anschauung, dafs die meisten africanischen Thiere eine weite Verbreitung haben. Dem gegenüber ist auf's Neue zu betonen, dafs verhältnifsmälsig sehr wenige Arten zugleich in West- und Ostafrica vorkommen, und wenn man bis jetzt viele derselben anführt, so beruht dies auf Täuschung in Folge mangelnder Aufmerksamkeit. Ein neues Beispiel bietet die Ootheca mutabilis Sahlb. Ueber ihre Verbreitung lesen wir in Kolbe, Stuhlmann Ostafrica [4] p. 338: Usambara, Buessa, Guinea, Sierra Leone, Togo, Alt-Calabar. Richtig sind nur die westafricanischen Fundorte an der Küste von Guinea; die ostafricanischen Ex. gehören anderen Arten an, von denen ich hier einige, wovon ausreichendes Material vorliegt, besprechen will.

a) Ootheca mutabilis: Testacea, supra laete ferrugineo-rufa, elytris concoloribus, vel basi, vel omnino nigris, angulis anticis prothoracis rotundatim dilatatis, parum prominulis. - Long. 5,5-7 mill. Guinea.

Femina: segmento quinto abdominali apice arcuato.

Die Art ist an den einfarbig bräunlich gelben Fühlern und Beinen, an denen die Schienen und Tarsen selten und dann nur schwach angedunkelt sind, sowie an den nicht oder wenig nach aufsen vortretenden, abgerundeten Vorderecken des Halssch. zu erkennen. Der Penis bildet eine etwas flachgedrückte Röhrf, welche sich von der Basis aus nach der Spitze allmählich sehr schwach verengt, am Ende ziemlich schnell gerundet-verschmälert und in eine kleine, schmale und stumpfe Spitze verlängert ist. Die Oeffnung ist lang, ihre Klappe pergamentbäutig, neben jedem Aufsenrande von einer geraden, stäbchenförmigen Chitinrippe durchzogen. 
b) Ootheca orientalis: Minor, rufo-testacea, supra saturatiore, saepe elytris nigris, singulo testaceo-rufo cincto; aut elytris omnino nigris, antennis articulis ultimis, tibiis tarsisque infuscatis, angulis anticis prothoracis angulatim dilatatis, prominulis. - Long. 5-6 mill.

Zanzibar, Usambara.

Femina: segmento quinto ventrali apice arcuato.

Den kleinen Ex. der vorigen ähnlich, unterseits, sowie auf Kopf und Halssch. etwas gesättigter roth gefärbt, namentlich in den dunklen Formen, die 2 oder 3 letzten Fühlerglieder nebst dem grölsten Theile der Schienen und die Tarsen angedunkelt. Die Vorderecken des Halssch. etwas kleiner, aber in den meisten Fällen an den Seiten winkelig heraustretend. Das 우 hat dieselbe Bildung des 5. Bauchringes wie die vorige; der Penis des $\sigma^{x}$ ist kurz und dick, mit mäfsig grofser Oeffnung, neben der die Seiten nach hinten fast gradlinig in eine scharfe Spitze verengt sind. Die Klappe ist kurz, pergamenthäutig, mit 2 breiten, mälsig weit getrennten, hinten convergirenden und an der Spitze sich berührenden Längsrippen, von denen jede am Ende höckerförmig aufwärts gebogen ist.

Ohne Berücksichtigung des Vaterlandes und der Penisbildung dürfte es schwer sein, die vorliegende Art von mutabilis zu trennen.

c) Ootheca Bennigseni: Rufo-testacea, elytris abdomineque dilutioribus, aut elytris nigris, fronte prothoraceque brunneo-rufis, antennis (articulis 4 vel 5 basalibus exceptis) tibiis (basi excepta) tarsisque nigris; angulis prothoracis parum prominulis. - Long. 5,3-7 mill.

Dar-es-Salaam, Pangani (v. Bennigsen), Zambesi.

Femina: segmento quinto abdominali apice utrinque sinuato, medio arcuatim producto.

Gröfser als die vorige, der mutabilis täuschend ähnlich, die Fühler vom 5. oder 6. Gliede an, die Schienen, mit Ausnahme der Basis, und die Tarsen schwarz. Das $q$ auf den ersten Blick an der Bildung des 5. Bauchringes zu erkennen, dessen Hinterrand nicht in einem gleichmälsigen Bogen abgerundet ist, wie in den beiden vorhergehenden Arten, sondern jederseits eine ziemlich tiefe bogenförmige Ausrandung besitzt, wodurch der mittlere Theil bedeutend nach hinten vorgezogen erscheint. Um das $\sigma^{-1} \mathbf{z u}$ erkennen, mufs man den Penis heraus präpariren, welcher von denen der andern Arten ungemein abweicht. Er ist anfangs an den Seiten parallel, erweitert sich leicht am Beginn der grofsen Oeffnung und ist in der Endbälfte derselben allmählich in schwacher Rundung in eine Spitze verengt, welche durch einen langen und mäfsig 
breiten, an der Basis gerundeten Spalt in zwei sehr scharfe Spitzen getheilt wird. Die Klappe der Oeffnung hat jederseits eine dicke und hohe Längsrippe, die sich über das Ende der Klappe hinaus als freier Stab bis an die Penisspitzen fortsetzt. Diese beiden Stäbe liegen dicht über dem Seitenrande des Penis, sind ähnlich wie dieser sanft gebogen, aber ihre Spitze krümmt sich am Ende nach aufsen.

Ich widme diese interessante Art Hrn. Gouverneur v. Bennigsen in Bismarckhöhe, welcher die Entomologie in Deutsch-Ostafrica in hohem Grade gefördert hat.

d) Ootheca longula: Subelongata, fere parallela, modice convexa, testaceo-albida, supra crebre subtiliter punctata, sat nitida, antennis (basi excepta), tibiis tarsisque infuscatis, angulis anticis prothoracis parvis, cylindrico-prominulis. - Long. 4,5-5,5 mill.

Bagamoyo (Kunow).

Bedeutend gestreckter, weniger gewölbt, feiner und dichter punktirt als die vorigen Arten, an den Seiten mehr parallel; weifslich gelb, das Halssch. mit Spuren von 5 etwas dunkleren, bräunlich durchscheinenden Makeln: 2 vor der Mitte, 3 dicht hinter derselben in einer Querreihe, die mittelste von diesen klein, punktförmig, die beiden andern gröfser. Die Vorderecken des Halssch. sind klein und bilden einen sehr kurzen, dicken Cylinder, auf dem die grofse Borstenpore eingestochen ist. Die Punktirung des Halssch. und der Fld. besteht aus ziemlich dicht gestellten feinen Punkten und Pünktchen in den $Z_{\text {wischenräumen. }}$

10. Oides flavipennis: Nigra, capite prothoraceque nitidis, hoc inaequaliter subtilius punctato, elytris stramineis vel testaceo-flavis, alutaceis, nitidulis, crebre subtiliter punctatis, singulo angustissime nigro-circumcincto, apice emarginato angulo suturali acuto. - Long. $11,5-14,5$ mill.

Montes Ukami (v. Bennigsen).

Femina: elytro singulo carinis tribus abbreviatis instructo.

Sehr breit eiförmig, schwarz, auf Kopf und Halssch. glänzend, unterseits matter, die Fld. strohgelb oder blafs bräunlich gelb, jede einzelne ringsum sehr fein schwarz gerandet. Kopf kaum deutlich punktirt, Fühler schlank, Halssch. doppelt so breit als lang, vorn schmaler als hinten, an den Seiten stark gerundet. Die Mitte der Scheibe gewölbt, ein Streifen am Vorderrande etwas vertieft, flach gedrückt, der Seitenrand durch einen starken Eindruck breit abgesetzt, vor dem Schildchen eine weite Vertiefung. Die Oberfläche ist ungleichmälsig, an einigen Stellen dichter, an 
andern sparsam, bald feiner, bald stärker punktirt, äufserst zart und nur unter starker Vergröfserung deutlich gewirkt. Schildchen gewirkt, punktirt, am Vorderrande fast glatt. Fld. gewirkt, matter als das Halssch., dicht und fein punktirt, jede am Hinterrande bogenförmig ausgeschnitten, sodafs die sehr spitze Nahtecke merklich nach hinten ausgezogen ist.

Das $\sigma^{\lambda}$ ist kleiner, kürzer gebaut als das 우, auf den Fld. ohne Vertiefungen und Rippen, der fünfte Bauchring in der Mitte mit einer grofsen, dreieckigen, flachen Grube, die am Vorderrande schmal beginnt und hinten den durch einen breiten, mälsig tiefen Einschnitt jederseits gebildeten Mittelzipfel einnimmt, dessen Hinterrand abgestutzt ist. Penis schlank, hell rostroth, zugespitzt.

Das $q$ hat auf jeder Fld. drei kurze Längsleisten, zwei innen, im vorderen Drittel, durch eine breite Mulde getrennt, die dritte aufsen, im mittleren Drittel der Länge; der fünfte Bauchring ist fast gleichmälsig gewölbt, am Hinterrande gerundet.

\section{Therpis n. gen.}

Corpus ovatum, minus conxexum, supra glabrum, punctatissimum, subtus cinereo-pubescens. Acetabula antica aperta. Antennae crassiusculae, subfiliformes, articulo $2^{0}$ parvo, articulis $3-5$ elongatis, reliquis crassioribus, brevioribus. Prothorax brevis, transversim convexus, antrorsum parum compresso-angusatus, foveis setigeris sat magnis, angulis anticis conico-prominulis; scutellum triangulare apice truncatum. Elytra prothorace parum latiora, apicem versus nonnihil ampliata, postice conjunctim rotundata, dorso minus convexa, callo humerali haud discreto, deplanato, fere nullo, epipleuris sat latis pone humeros sensim angustatis, ante apicem oblitteratis.

Diese Gattung erinnert am meisten an Aphophylia, ist aber durch die gezähnten Klauen und die unbespornten Vorderschienen verschieden. Der Körper ist schwach gewölbt, vorn schmal und erweitert sich in schwacher Rundung nach hinten, wo die Fld. gemeinschaftlich ziemlich breit abgerundet sind. Mundtheile, Kopfschild, Augen und Stirn baben die normale Bildung der meisten Galerucinen, die Fühler sind mäfsig lang und stark, reichen beim $\rho$ nicht bis zur Mitte der Fld, beim $\sigma^{\lambda}$ darüber hinaus. Glied 1 keulenförmig, so lang als das Auge, 2 kurz, wenig länger als breit, 3 und 4 gestreckt, an der Spitze verdickt, jedes etwa so lang als 1, 5 ähnlich wie 4, nur kürzer, die folgenden sind merklich dicker und nehmen nach dem Endgliede hin allmählich an Länge, die beiden letzten Glieder auch an Breite ab. Die Stirn ist über den oben undeutlich begrenzten grofsen und 
flachen" Höckern dicht punktirt, ähnlich wie das Halssch. Dieses ist $2 \frac{1}{2}$ mal so breit als lang, vorn gerade abgeschnitten, hinten fast unmerklich gerundet, schwach gewölbt, aber an den Seiten stark abfallend, nach vorn hin zusammengedrückt, von oben betrachtet also nach vorn verengt, in jeder Ecke mit grofser Pore und langer Tastborste. Das Schildchen ist klein, gleichseitig-dreieckig, vorn flach, hinten gewölbt und an der Spitze schmal ahgestutzt. Fld. in den Schultern kaum breiter als das Halssch., schwach gewölbt, äufserst dicht punktirt, die Punkte scharf eingestochen, wenig gröfser, aber tiefer als die des Halsschildes, mit zarten, leistenförmigen $\mathrm{Zwischenräumen;} \mathrm{der} \mathrm{Seitenrand} \mathrm{schmal} \mathrm{abgesetzt,} \mathrm{vor}$ der Mitte etwas ausgebreitet, die Schulterbeule flach gedrückt, vorn und innen durch einen weiten Eindruck kaum merklich abgesetzt, die Epipleuren vorn ziemlich breit, hinter der Schulter ganz allmählich verengt und an der hinteren Aufsenecke erlöschend. Schienen auf dem Rücken mit kahlem Längsstreifen, der eine schwache Mittelleiste besitzt, an der Spitze der 4 Hinterschienen ein kurzer, jedoch ziemlich starker Dorn. Erstes Tarsenglied fast so lang als die beiden folgenden zusammen, das dritte kürzer als das zweite, an der Spitze tief ausgerandet, das vierte doppelt so lang, Klauen an der Basis breit, in der Mitte in einen nach innen gebogenen starken und spitzen Zahn ausgezogen, der etwas kürzer als der feinere Endtheil ist. Beim $q$ ist der fünfte Bauchring gleichmäfsig gewölbt und in der Mitte des Hinterrandes gerade abgestutzt, beim $\sigma^{\top}$ hat derselbe hinter der Mitte einen allmählich verbreiterten und vertieften, bogenförmigen Längseindruck und am Hinterrande einen starken, gerundeten Ausschnitt.

Therpis smaragdina: Argillacea vel testacea, antennis articulis sex vel septem ultimis mandibulisque apice piceis, elytris saturate smaragdinis, creberrime punctatis, subopacis, limbo laterali testaceo. - Long. $7\left(\sigma^{\top}\right)-8($ ( ) $)$ mill.

Mombas (Schneider).

Oben ziemlich matt, unten etwas glänzender, weifslich, thonfarbig oder, bei weniger gut gehaltenen Exemplaren, blafs röthlich gelbbraun, die Endhälfte der Mandibeln pechbraun, die 6 oder 7 letzten Glieder der Fühler pechschwarz bis schwarz; Fld. gesättigt smaragdgrün, äufserst dicht punktirt, ein Seitensaum röthlich gelbbraun. Dieser Saum nimmt in der Mitte der Fld. ungefähr $\frac{1}{6}$ der Breite ein, ist gleich breit, nur vorn, unterhalb der flach gedrückten Schulterbeule verschmälert, in der inneren Hälfte sparsamer als der übrige Theil der Fld. punktirt, in der äufseren nur fein gerunzelt. 
Platyxantha costatipennis Jac. Transact. $1895,335=$ Apophylia costipennis Fairm., C. R. Soc. Belg. 1891, $304=M e-$ galognatha Haroldi Fairm. Ann. Fr. 1887, 365 = Luperus elegans Harold, Stett. Z. 1879, 336. Das Thier ist als Platyxantha elegans Har. zu führen.

Agelasa fulvicollis Quedenf. Berl. Z. 1891, 174 ist das 9 von Malacosoma nigrum All. Ann. Belg. 1889, C. R. 69. Allard hat l. c. das $\sigma^{\pi}$ beschrieben, welches kleiner und schlanker als das ㅇ, 7,5-8 mill. lang und auf jeder Fld. hinter dem Schildchen an der Nabt mit einer Grube versehen ist. Ich besitze Männchen von Zanzibar (Giesbers) und Weibchen, 10 mill. lang, von Mikindani (Schneider). Das Thier ist keine Malacosoma (denn die vorderen Hüftpfannen geschlossen, Schildchen zugespitzt, Ränder des Halsschildes kahl), sondern ein Luperodes.

12. Mombasica magna: Subcylindrica, sat dense subtilissimeque fulvo-griseo pubescens, obscure viridi acnea, parum nitida, elytris aurichalceo-micantibus, fere opacis, ore, antennis tarsisque nigris; capite dense ruguloso-punctato, tuberculis frontalibus magnis, alutaceis, sericeo-micantibus, prothorace parum transcerso crebre punctato, nitidulo, linea media impresso, lateribus deflexis medio dente magno, acuto ( $\left.\zeta^{\top}\right)$, vel parvo, obtuso (ㅇ) armatis, scutello semiovato, nigro, dense punctato et pubescente, linea media obsolete impresso; elytris creberrime punctatis, interstitiis angustissimis punctulato-rugulosis. - Long. 12-14,5 mill.

Mombas (Schneider).

Mas: Fronte magna, transversim quadrata, antennis corpore parum brevioribus, tibiis anticis margine interno pone medium valde rotundatim dilatatis, segmento quints abdominali longitudinaliter sulcato, apice truncato in medio parum profunde emarginato.

Femina: Robustior, fronte brevi, antennis brevioribus, tibiis anticis pone medium parum crassioribus, segmento $5^{0}$ abdominali haud impresso, apice leniter rotundato.

Ich besitze nur Momb. subinermis, welche Fairmaire ,extrèmement voisine" der armicollis nennt, daher kann die vorliegende Art mit letzterer nicht identisch sein; sie ist viel gröfser, namentlich breiter, durchgängig stärker punktirt und bietet auch in der Körperfarbe bei den mir vorliegenden Stücken nicht den geringsten Uebergang. Annähernd cylindrisch $\left(\sigma^{\top}\right)$, oder die Fld. hinter dem ersten Drittel der Länge deutlich verbreitert (ㅇ), unten sehr dunkel metallisch grün, Mund, Fühler, Schienen und Tarsen schwarz, Ober- 
lippe oft pechbraun; Oberseite lebhafter metallisch grün, die Fld. mit Bronceschimmer. Der ganze Körper ziemlich dicht, aber sehr fein und kurz grau behaart, die Härchen in gewisser Richtung gelblich. Kopf mit langem Halse und längerer, quer viereckiger $\left(\zeta^{\top}\right)$ oder kurzer Stirn (q), letztere dicht runzelig punktirt, die grofsen Stirnbeulen nur sparsam fein punktirt, aber dicht gewirkt, matt, zuweilen mit bläulichem Anfluge. Halssch., von oben betrachtet, fast so lang als breit, mälsig dicht punktirt, ziemlich glänzend $\left(\zeta^{\star}\right)$, oder deutlich quer, etwas dichter punktirt und schwächer glänzend ( $(q)$, mit durchgehender, wenig tiefer Mittelrinne; die herabgebogenen Seiten in der Mitte stumpfwinklig erweitert (ㅇ), oder mit einem grofsen, ziemlich scharfen, wagerecht alstehenden Zahne. Fld. äufserst dicht punktirt, die Mehrzahl der schmalen $Z$ wischenräume mit einer feinen Querwurzel.

Beim $\widehat{\jmath}$ sind die Fühler bedeutend länger als beim 오, der Inneurand der Vorderschienen ist in der Spitzenhälfte sehr stark bogenförnig erweitert, der 5 . Bauchring hat eine breite Mittelfurche, die vorn flacher, glänzender, hinten breiter und tiefer ist, so dass ihre Ränder hinten eine hohe, wulstartige Ecke bilden, die sehr dicht behaart ist. Der Hinterrand ist abgestutzt und in der Mitte sanft ausgerandet.

Cerathispa Ws. Deutsch. Z. 1900, 213 ändere ich wegen der gleichnamigen Gattung von Gestro in Dorcathispa um, ebenso Psecas, Archiv 1899, I, 3, p. 272, in Psecadia, ferner Scymnomorphus Ws. D. Z. 1897, 303 wegen Scymnomorpha Blackburn, Trans. Reg. Soc. South Australia 1892 in Scotoscymnus, endlich Cassida Kraatzi m. Archiv f. Naturg. 1900, I, 2, 295 in C. Spaethi.

13. Cladocera variegata: Subcylindrica, subtus rufo testacea, pleuris sternalibus infuscatis, genubus dorsoque tibiarum et tarsorum nigris, antennis nigris, capite flavo-testaceo, mandibulis, macula transversa inter antennas verticeque piceis, prothorace crebre punctulato, flavo-albido, maculis quinque nigris, scutello elytrisque flavo-albidis, illo interdum infuscato, his subtilissime alutaceis, crebre punctatis, macula communi maculisque binis vel tribus ante medium fasciisque duabus pone medium brunneis, obscurior marginatis. Long. 11-13,5 mill.

Mombas (Schneider).

Var. a) Macula interiore cum macula communi fasciisque inter se confluentibus. 


\section{$2 \mathrm{BHL}$ Biodiversity Heritage Library}

Weise, Julius. 1901. "Beschreibungen africanischer Chrysomeliden nebst synonymischen Bemerkungen." Deutsche entomologische Zeitschrift 1900, 446-459.

View This Item Online: https://www.biodiversitylibrary.org/item/103695

Permalink: https://www.biodiversitylibrary.org/partpdf/245187

\section{Holding Institution}

Harvard University, Museum of Comparative Zoology, Ernst Mayr Library

\section{Sponsored by}

Harvard University, Museum of Comparative Zoology, Ernst Mayr Library

\section{Copyright \& Reuse}

Copyright Status: Public domain. The BHL considers that this work is no longer under copyright protection.

This document was created from content at the Biodiversity Heritage Library, the world's largest open access digital library for biodiversity literature and archives. Visit BHL at https://www.biodiversitylibrary.org. 